\title{
BMJ Open A double blind, randomised, placebo- controlled trial to evaluate the efficacy of metformin to treat preterm pre- eclampsia (PI2 Trial): study protocol
}

\author{
Catherine Cluver, ${ }^{\oplus 1,2}$ Susan P Walker, ${ }^{2,3}$ Ben W Mol, ${ }^{4}$ David Hall, ${ }^{5}$ \\ Richard Hiscock, ${ }^{6}$ Fiona C Brownfoot, ${ }^{2,3}$ Tu'uhevaha J Kaitu'u-Lino,, \\ Stephen Tong ${ }^{2,3}$
}

To cite: Cluver C, Walker SP, Mol BW, et al. A double blind, randomised, placebo-controlled trial to evaluate the efficacy of metformin to treat preterm pre-eclampsia (PI2 Trial): study protocol. BMJ Open 2019;9:e025809. doi:10.1136/ bmjopen-2018-025809

- Prepublication history and additional material for this paper are available online. To view these files, please visit the journal online (http://dx.doi org/10.1136/bmjopen-2018025809).

Received 2 August 2018 Revised 26 February 2019 Accepted 27 February 2019

Check for updates

(C) Author(s) (or their employer(s)) 2019. Re-use permitted under CC BY-NC. No commercial re-use. See rights and permissions. Published by BMJ.

For numbered affiliations see end of article.

Correspondence to Dr Catherine Cluver; cathycluver@hotmail.com

\section{ABSTRACT}

Introduction Pre-eclampsia is a major complication of pregnancy, globally responsible for 60000 maternal deaths per year, and far more fetal losses. There is no definitive treatment other than delivery. A therapeutic that could quench the disease process would be useful to treat preterm pre-eclampsia, as it could allow these pregnancies to safely continue to a gestation where fetal outcomes are significantly improved. We have published preclinical data to show that metformin, a drug known to be safe in pregnancy and commonly used to treat gestational diabetes, has potent biological effects making it another promising candidate to treat pre-eclampsia. Here, we describe a phase II clinical trial to examine whether administering extended-release metformin may be effective in treating women with preterm pre-eclampsia (PI2 Trial).

Methods The PI2 Trial is a phase II, double blind, randomised controlled trial that aims to recruit 150 women with preterm pre-eclampsia (gestational age $26+0$ to $31+6$ weeks) who are being managed expectantly. Participants will be randomised to receive either $3 \mathrm{~g}$ of metformin or placebo daily. The primary outcome is time from randomisation until delivery. A delay in delivery of 5 days is assumed to be clinically relevant. The secondary outcomes will be a maternal composite and neonatal composite outcome. All other outcomes will be exploratory. We will record adverse events.

Ethics and dissemination This study has ethical approval (Protocol number M16/09/037 Federal Wide Assurance Number 00001372, Institutional Review Board Number IRB0005239), is registered with the Pan African Clinical Trial Registry (PACTR201608001752102) and the South African Medicine Control Council (20170322). Data will be presented at international conferences and published in peer-reviewed journals.

Trial registration number PACTR201608001752102; Pre-results.

\section{INTRODUCTION}

Pre-eclampsia is one of the most serious complications of pregnancy affecting $5 \%$ of pregnancies worldwide. ${ }^{1}$ It is a multisystem

\section{Strengths and limitation of this study}

- This is a protocol for a randomised, double blind, placebo-controlled clinical trial.

- This is the first trial to assess whether metformin is a treatment option for pre-eclampsia.

- We plan to recruit 150 participants which provides sufficient power for our primary outcome (time from randomisation to delivery).

- Our trial may be underpowered to show improvements in maternal and perinatal outcomes.

- If the trial yields a positive result, larger multicentre studies will be needed.

disorder involving maternal vessels (causing hypertension and endothelial dysfunction), the kidneys, the liver, the lungs, the haematological system, the cardiovascular system and the fetoplacental unit. ${ }^{2}$ In its most severe form, it affects the brain, causing seizures (eclampsia), cerebrovascular events and even death.

It is a leading cause of maternal and fetal/neonatal morbidity. ${ }^{3}$ Globally, pre-eclampsia is responsible for $>60000$ maternal deaths annually ${ }^{4}$ and it is estimated that for every pre-eclampsia related death there are 50 to 100 other women who experience significant morbidity associated with pre-eclampsia. ${ }^{5}$

At present, the only treatment available for pre-eclampsia is termination/delivery of the pregnancy. This poses a difficult clinical dilemma in the setting of preterm pre-eclampsia. Clinicians are often forced to deliver early to prevent disease progression and major maternal morbidity (ie, severe maternal organ injury), but in doing so, inflict severe prematurity on the fetus. In particular, fetuses delivered at less than 33 weeks' gestation are at significant risk of severe disability 
including cerebral palsy, stroke (intracerebral bleeding), retinopathy of prematurity, chronic lung disease and death. $^{6}$

We have recently completed a phase II, double blind, randomised controlled trial, the Pre-eclampsia Intervention with Esomeprazole (PIE) trial, to evaluate whether $40 \mathrm{mg}$ of esomeprazole was a possible treatment for early onset pre-eclampsia. This trial showed no between-group differences in median time from randomisation to delivery (11.4 days in the esomeprazole group and 8.3 days in the placebo group [95\% CI -2.9 to $8 \cdot 8, \mathrm{p}=0.31]) .{ }^{78}$ Here, we propose metformin as the next therapeutic candidate to test in a clinical trial for preterm pre-eclampsia.

Metformin is a promising therapeutic candidate as it has been shown to reduce soluble fms-like tyrosine kinase 1 (sFlt-1) and soluble endoglin (sEng) secretion from endothelial cells and primary trophoblasts. ${ }^{9}$ These key anti-angiogenic molecules released from the placenta are responsible for the widespread endothelial dysfunction and impaired vascular relaxation observed in pre-eclampsia. Reduced angiogenesis is also thought to contribute to placental hypoxia and to the development of pre-eclampsia. Metformin has also been shown to improve endothelial dysfunction, improve blood vessel relaxation and promote angiogenesis. ${ }^{9}$ Given these multiple potential effects, metformin is a particularly promising therapeutic candidate.

\section{OBJECTIVES}

The primary objective is to examine whether $3 \mathrm{~g}$ of metformin XR can safely prolong gestation among women with preterm pre-eclampsia who are being managed expectantly, compared with expectant management alone. A prolongation of 5 or more days will be considered clinically significant.

The secondary objectives are to examine whether metformin can improve maternal and neonatal composite outcomes.

\section{METHODS}

The full protocol is included as supplementary information (online supplementary file 1).

\section{Study design}

Phase II hospital based, double blind, randomised, placebo-controlled trial.

\section{Study population}

Pregnant women diagnosed with pre-eclampsia at a gestational age between $26+0$ weeks to $31+6$ weeks at Tygerberg Hospital (Western Cape Province of South Africa) will be invited to participate. To be eligible for this study the treating clinicians need to have made an initial assessment and deemed that the patient is suitable for expectant management and delivery is not immediately required or anticipated in the next 48 hours.
Box 1 Inclusion criteria

A diagnosis of one of the following
Pre-eclampsia
Gestational hypertension with evidence of pre-eclampsia
Preexisting hypertension with evidence of pre-eclampsia
Unclassified proteinuric hypertension
AND all of the following is present
Gestational age between $26+0$ weeks and $31+6$ weeks
Estimated fetal weight by ultrasound between $500 \mathrm{~g}$ and $1800 \mathrm{~g}$ (if
gestation is not certain)
Singleton pregnancy
The managing clinicians have made the assessment to proceed
with expectant management and that delivery is not expected with-
in 48 hours
The managing clinician and neonatologist believe that the fetus
could potentially be delivered in a viable condition
No suspicions of a major fetal anomaly or malformation.
The patient will be admitted to hospital for expectant management
and standardised care

\section{Inclusion criteria}

We will recruit women with a singleton pregnancy diagnosed with pre-eclampsia and chronic hypertension with superimposed pre-eclampsia defined according to the criteria published by The International Society for the Study of Hypertension In Pregnancy, ${ }^{10}$ and require the presence of significant proteinuria (more than $300 \mathrm{mg}$ in a 24 hours urine collection). Some women only present for antenatal care after 20 weeks gestation. We will include these women and classify these women as unclassified proteinuric hypertension during the pregnancy according to the classification of Davey and MacGillivray. ${ }^{11}$ Postpartum, these women will be reclassified as having had pre-eclampsia or chronic hypertension based on their blood pressure and need for medication more than 6 weeks after the due date.

Gestational age will be determined by either period dates (if the woman is certain of her last menstrual period) or by an early or mid trimester pregnancy ultrasound. If the gestational age is uncertain, we will recruit participants with an estimated fetal weight between 500 and $1800 \mathrm{~g}$, determined by ultrasound performed at presentation. A full list of inclusion criteria is provided in box 1 .

\section{Exclusion criteria}

Exclusion criteria include women with established maternal or fetal compromise that necessitates delivery within 48 hours, the current use of metformin, contraindications to the use of metformin or the use of medications that interact with metformin. A full list of exclusion criteria is provided in box 2 .

\section{Participant enrolment}

Participants will be identified after they have been admitted to the tertiary referral centre with a diagnosis of preterm pre-eclampsia for expectant management. An 


\section{Box 2 Exclusion criteria}

\section{Any of the following at the initial assessment}

- The patient is unable or unwilling to give consent

- Established fetal compromise that necessitates delivery

- The presence of: eclampsia

a. Severe hypertension, defined as a systolic blood pressure greater than or equal to $160 \mathrm{~mm} \mathrm{Hg}$ or diastolic blood pressure greater than or equal to $110 \mathrm{~mm} \mathrm{Hg}$ that cannot be controlled with antihypertensive medication within 48 hours of admission

b. Cerebrovascular event

c. Posterior reversible encephalopathy syndrome

d. Severe renal impairment, defined as a creatinine level $\geq 125$ $\mu \mathrm{mol} / \mathrm{l}$ or a need for dialysis

e. Pulmonary oedema

f. Left-sided heart failure

g. Disseminated intravascular coagulation

h. Platelet count $<50 \times 10^{9}$

i. Haemolysis, elevated liver enzymes and low platelets syndrome

j. Liver transaminases $>500 \mathrm{IU} / \mathrm{L}$

k. Liver haematoma or rupture

I. Severe ascites

Diabetes

- Current use of metformin

- Contraindications or a hypersensitivity reaction to the use of metformin

Current use of a drug that may be affected by metformin

information leaflet will be given to all potential participants and written informed consent will be obtained (online supplementary file 2 and 3 ).

\section{Randomisation and allocation concealment}

After informed consent, participants will be randomised to metformin or placebo in a 1:1 ratio. An online, web-based sequence generator system linked with codes for placebo and treatment tablets will be used. Both researchers and participants will be blinded.

The gestational age at diagnosis is likely to affect allowable length of pregnancy prolongation. To ensure treatment group allocation is balanced for this potentially confounding variable, we will stratify by gestational age. Strata 1 includes women with pregnancies of gestational age of $26+0$ up to and including $28+6$ weeks (500 to $1200 \mathrm{~g}$ if gestation is unknown). Strata 2 includes women with pregnancies of gestational age $29+0$ up to and including $31+6$ weeks ( 1200 to $1800 \mathrm{~g}$ if gestation is unknown). Thus, randomisation will include blocking within each gestational age stratum. We will use blocks of four to six with the size and order randomly assigned.

Once the participants have been randomised, the treatment pack with the same code will be allocated to the participant. All treatment packs will be identical and will contain either active tablets or placebo. The researchers will have no access to the randomisation list. This process will ensure that there is allocation concealment throughout the conduct of the trial.

Each participant will be given an individual treatment pack containing either $500 \mathrm{mg}$ of metformin XR or identical placebo tablets which have been produced by Merck (http://www.merck.com/index.html). Labelling, storage and preparation will be done according to the requirements of the Medicines for Human Use (Clinical Trials) regulations. Participants will be started on six tablets daily in divided doses. If side effects develop the dose will be decreased until the side effects improve and then increased again if tolerated.

\section{Co-interventions}

Participants will remain under the care of the hospital treating team and the study will not alter or interfere with the routine care provided for women with preterm pre-eclampsia, including the decision for delivery. Management for preterm pre-eclampsia involves admission to hospital with close maternal and fetal surveillance. Maternal surveillance includes four times an hour blood pressure measurement, two times per day clinical assessment, daily urinalysis and two times per week assessments with blood tests (full blood count, renal function tests and hepatocellular enzymes if haemolysis, elevated liver enzymes and low platelets syndrome is suspected) and 24 hours urinary protein measurement on admission. Fetal surveillance includes six times an hour cardiotocography and ultrasound assessments every 2 weeks (or more frequently if clinically indicated) for Doppler velocimetry of the umbilical artery, middle cerebral artery, ductus venosus, amniotic fluid volume assessment and fetal growth.

All participants will receive two doses of betamethasone 24 hours apart to reduce the risks of neonatal respiratory distress syndrome, intracranial haemorrhage and necrotising enterocolitis for the neonate. A single repeat dose will be given 1 week later as per hospital protocol. ${ }^{12}$ Most participants will be on antihypertensive treatment, and the dose and number of antihypertensives will be recorded. All women should already be receiving calcium, iron and folic acid supplementation.

Clinical care will be left up to the discretion of the clinical team. The indication for delivery will be a clinical decision. Indications for delivery may include inability to achieve blood pressure control, the development of major maternal or fetal complications or intrauterine fetal death. Expectant management will usually end at a gestation of 34 weeks.

\section{Sample collection}

Blood samples will be routinely collected two times a week. The routine blood samples include measurements of the haemoglobin, the platelet count and the urea and creatinine levels. These will be used by the managing clinicians to determine disease severity and may trigger delivery.

\section{Outcomes}

The primary outcome is to examine whether up to $3 \mathrm{~g}$ of metformin daily can safely prolong gestation in women with preterm pre-eclampsia diagnosed between $26+0$ to $31+6$ weeks gestation, compared with standard of care, 


\section{Box 3 Secondary Outcomes}

\section{Secondary outcomes}

Composite maternal outcome:

- Maternal death

- Eclampsia

- Pulmonary oedema

- Severe renal impairment or dialysis

- Cerebral vascular event

- Liver haematoma or rupture

- Placental abruption

Composite neonatal outcome:

- Neonatal death within 6 weeks after the due date

- Grade III or IV intraventricular haemorrhage

- Necrotising enterocolitis (diagnosed on radiological studies)

- Bronchopulmonary dysplasia (defined as needing oxygen at day 28 of life, either on a ventilator, by continuous positive airway pressure or via a nasal catheter)

expectant management alone. This is set in a superiority framework with two-sided hypothesis regions for all statistical testing. A prolongation of 5 days or longer will be considered clinically significant.

The secondary outcome is to determine whether metformin improves maternal composite and neonatal composite outcomes. The composite maternal outcome includes maternal death, eclampsia, pulmonary oedema, severe renal impairment or the need for dialysis, a cerebral vascular event, the development of a liver haematoma or rupture and placental abruption. The composite neonatal outcome will include neonatal death within 6 weeks after the due date, grade III or IV intraventricular haemorrhage defined enlarged ventricles associated with haemorrhage or haemorrhage extending into the cerebral tissue around the ventricles on imaging, necrotising enterocolitis defined on radiographic studies or bronchopulmonary dysplasia defined as needing oxygen at day 28 of life, either on a ventilator, by continuous positive airway pressure or via a nasal catheter. See box 3

All other outcomes, including outcomes nested within the composite secondary outcomes will be classified as exploratory outcomes. These are listed in box 4 .

\section{Withdrawal from the study}

All participants will be informed that they are free to withdraw from the study at any time, and that this will not affect their clinical care. In the event of withdrawal, we will seek the patient's permission to use their clinical information and laboratory samples collected thus far in subsequent analyses.

\section{Duration of the trial}

It is anticipated that the study will be completed in approximately 3 years (2018 to 2020). In our previous PIE study, we included 120 women in 15 months. ${ }^{8}$
Box 4 Exploratory outcomes

\section{Exploratory outcomes}

Maternal exploratory outcomes:

- Maternal death

- Eclampsia

- Pulmonary oedema

- Severe renal impairment or dialysis

- Cerebral vascular event

- Liver haematoma or rupture

- Abruptio placentae

- Admission to a high care or intensive care unit

- Posterior reversible encephalopathy syndrome

- Left ventricular failure

- Serum creatinine $>125 \mu \mathrm{mol} / \mathrm{l}$

- Proteinuria $>3 \mathrm{~g} / 24$ hours

- Severe hypertension

- Disseminated intravascular coagulation

- Platelet count less than $50 \times 10^{9}$

- Haemolysis, elevated liver enzymes and low platelets syndrome

- Liver transaminases $>500 \mathrm{lU} / \mathrm{L}$

- Placental abruption

- Mode of delivery

- Use of antihypertensive agents

- Major postpartum haemorrhage

- Thromboembolic disease

- Moderate or severe ascites

Fetal exploratory outcomes:

- Reversed a-wave in the ductus venosus on fetal ultrasound

- Significant changes in fetal heart rate patterns on the non-stress test that necessitate delivery

- Intrauterine fetal demise

- Fetal growth restriction at birth

- Persistent reversed flow in the umbilical artery

- Redistribution in the middle cerebral artery

Neonatal exploratory outcomes:

- Neonatal death within 6 weeks after the due date

- Grade III or IV intraventricular haemorrhage

- Necrotising enterocolitis

- Bronchopulmonary dysplasia

- APGAR score $<7$ at $5 \mathrm{~min}$

- Umbilical artery $\mathrm{PH}<7.05$

- Umbilical artery lactate

- Surfactant use

- Neonatal intensive care or special care unit admission

- Intubation and mechanical ventilation

- Continuous positive airway pressure support

- Grade III/IV hyaline membrane disease hospital stay

- Incidence of retinopathy of prematurity neonatal sepsis

\section{Confidentiality}

Patient confidentiality will be protected according to the regulations set forth by Stellenbosch University's Human Research Ethics Committee or Institutional Review Board.

\section{Data management and statistical analysis}

Data will be collected prospectively using a REDCap database. ${ }^{13}$ Data entry and checking will be continuous, and queries will be addressed contemporaneously to ensure clarification without delay. All data will be double checked for accuracy. 
The patient characteristics, by treatment group at randomisation, will be presented as mean (SD), median (25th to 75th percentile), minimum, maximum and count (\%) depending on type and distribution.

The primary outcome will be measured from the time of randomisation to delivery. Secondary maternal and neonatal outcomes will be measured from the time of recruitment until 6 weeks after the due date. The significance level for the primary outcome is set at 0.05 and all hypothesis testing will be two-sided. If, and only if, there is a significant difference for the primary outcome, the two secondary outcomes will be tested at a significance level of 0.025 . Standard 95\% CI will be used to present the analysis of the primary outcome. The width of the CI used will be adjusted for the secondary outcomes if tested. For raw results data, the primary outcome will be summarised using median (25th to 75 th percentile) along with minimum and maximum values.

All secondary outcomes and exploratory outcomes of inter-test will be presented by number $(\%)$ by treatment arm. Primary outcome effect size will be presented as difference in medians and 95\% CI, adjusted for gestational age strata based on the quantile regression model. $\mathrm{P}$ values will be reported associated with $95 \% \mathrm{CI}$ for the secondary outcomes if tested. Exploratory outcomes will be presented with $95 \%$ CI.

Modelling will use quantile regression to assess difference in median prolongation between groups. This will be supplemented by survival analysis (ie, time until delivery), using Cox proportional hazards regression $(\mathrm{CPH})$ and Kaplan-Meier survivorship curves. Survival outcomes will not be subject to hypothesis testing. For both quantile regression and survival analysis effect estimates will be presented as group difference with $95 \%$ CI.

The number of events for each of the composite binary outcomes is likely to be small. An exact logistic regression model containing treatment group and gestational age strata will be used. In the event that it is not possible to obtain estimates, due to small counts each hypothesis will be tested, unadjusted for gestational strata, using Fisher's exact test. Point estimates of percentage and exact 95\% CI will also be provided.

For the primary outcome a covariate adjusted analysis with both treatment group and gestational age designbased strata modelled as fixed will constitute the primary analysis outcome. Unadjusted and adjusted CPH analysis are only supplemental to this primary analysis. For the secondary outcomes composite binary outcomes will be modelled using a logistic regression model containing both treatment group and gestation aged based strata.

The sensitivity analyses that will be conducted on the primary outcome are; first, the treatment received; second, gestational age as an effect modifier; third, covariate adjusted for an estimated fetal weight less than the $10^{\text {th }}$ and $3^{\text {rd }}$ centile on ultrasound at the time of randomisation and absent umbilical artery end diastolic flow as these are more likely to be associated with shorter randomisation to delivery times and lastly, a survival analysis will be performed using CPH adjusted for gestational age strata and Kaplan-Meier survivorship curves.

No sensitivity analysis for primary outcome outcomes is planned for missing data as we expect these mothers not to have missing outcome data or baseline measured pre-treatment covariates. If the neonate is lost to follow-up the outcome will be set to missing, a sensitivity analysis will be performed to assess strength of inference given pattern of missingness by setting missing values to no event, mean event rate or event to assess dependence on inference on the unknown outcomes.

Statistical software used is Stata V.15 (StataCorp. 2017. Stata Statistical Software: Release 15). The trial presentation will meet the CONSORT statement requirements (http://www.consort-statement.org/).

\section{Sample size and stratification according to gestation at recruitment}

The primary research question is whether metformin will result in a gain in the median length of gestation compared with placebo therapy. A prolongation of more than 5 days will be considered clinically significant. Given the focus on median prolongation and the skewed nature of the gestation prolongation (seen in the two trials listed below) we based sample size calculations on the geometric mean ratio. Analysis was performed both using PASS 13 (Power Analysis and Sample Size Software [2014]. NCSS, LLC.) and the Powercal programme running in Stata V.15. ${ }^{14}$

We have two sources for data on the prolongation of gestation in pre-eclamptic mothers receiving expectant management which were both performed at the same hospital where we plan to run this trial (Tygerberg Hospital). Hall et al, in the largest descriptive study on expectant management of preterm pre-eclampsia, reported a mean gestation prolongation of $11(\mathrm{SD} 7)$ days equivalent to a coefficient of variation $(\mathrm{CV})=0.64,{ }^{15}$ and the PIE trial, ${ }^{8}$ where patients randomised to the placebo arm had an overall mean prolongation of 13.1 (SD 12.2) days, a CV $=0.93 .{ }^{8}$ In this study for mothers entering at gestations greater than 29 weeks, the $\mathrm{CV}=0.8$. We have therefore chosen to use a CV $=1.0$, slightly larger than the 0.93 found in our PIE study, to allow for adequate power if greater variability in prolongation times occurs in PI2 Trial.

For $90 \%$ power, with a two-sided alpha set at 0.05 , control median prolongation of 8.3 days and prolongation of gestation of 5 days with the measure of variability used in this analysis being the $\mathrm{CV}=($ data $\mathrm{SD}) /$ data mean $)$, we require 65 participants per group. Allowing for 10 dropouts per group we require 75 participants per arm and therefore a total of 150 participants.

The gestational age at diagnosis is likely to affect allowable length of pregnancy prolongation. For instance, those diagnosed at $31+6$ will be delivered at 34 weeks. Thus, such patients will have a maximum length of pregnancy prolongation of 15 days. In contrast, those diagnosed at 28 could conceivably obtain many weeks of pregnancy 
prolongation. Further it is possible that metformin efficacy may vary with gestational age at diagnosis. To ensure treatment group allocation is balanced for this potential variable, we will stratify randomisation into strata based on gestational age. Stratum 1 will include pregnancies of a gestational age of 26 weeks 0 days up to and including 28 weeks and 6 days. Stratum 2 will include pregnancies from 29 weeks and 0 days up to and including 31 weeks and 6 days.

More detail is available in the trial statistical analysis plan (online supplementary file 4).

\section{Adverse events}

Reporting and handling of adverse events and serious adverse will be in accordance with the good clinical practice guidelines.

\section{Unblinding}

Given that the safety profile of metformin in pregnancy is well established, we anticipate the need for unblinding to be unlikely. However, we will have the following procedures in place should unblinding be required. Sheets with lists of 25 randomisation codes each will be sealed in individual, signed, numbered envelopes. If unblinding is requested, the relevant envelope will be opened by a person not involved in the trial in the presence of two witnesses, the group allocation read and the list re-sealed in an envelope and signed. The randomisation envelopes will be accessible at all times to the principal investigator who will be contactable by mobile telephone. The principal investigator will co-ordinate this process.

\section{Early termination of the trial}

If for any reason, there is a need to stop the trial prematurely this decision will be taken by the data and safety monitoring committee.

\section{Patient and public involvement}

Patients and the public were not involved in the development of the research question, the design of the study, the conduct or recruitment and the burden of the intervention was not assessed by the patients or the public. The results of the study will be emailed to the participants.

\section{DISCUSSION}

Pre-eclampsia is a serious life-threatening condition for both the mother and fetus and is associated with severe maternal and perinatal morbidity. If a treatment were to be discovered, it would have a major impact on both maternal and perinatal health. An ideal drug would ameliorate the biological disease process of pre-eclampsia in the mother and placenta, reduce the risk of serious complications from developing and allow pregnancies that were complicated by the disease at an early gestation to gain gestation without putting the mother and fetus at significantly increased risk.

Recently a number of clinical trials have been proposed for assessing novel treatments for pre-eclampsia, but only a few have been completed. Our group has recently completed the PIE trial assessing esomeprazole to treat preterm pre-eclampsia. $^{8}$ In this trial a daily dose of $40 \mathrm{mg}$ esomeprazole did not prolong gestation or have any effect on biomarkers associated with pre-eclampsia. Sildenafil was assessed in a single site, double blind, randomised controlled trial in Brazil. ${ }^{16}$ Over a 28 month period, 100 women were recruited and results were available for 93 women. There was a significant prolongation of gestation in the sildenafil group of 4 days, but given that sildenafil is a vasodilator it is possible that this prolongation in gestation may have occurred because the drug decreased blood pressure and mitigated a clinical reason to deliver, rather than temporising disease progression. Antithrombin was assessed to treat preterm pre-eclampsia in the PRESERVE-1 trial which enrolled 120 women from 23 tertiary hospitals over 28 months (ISRCTN23410175).${ }^{17}$ There was no difference in prolongation of pregnancy or composite neonatal outcomes. ${ }^{18}$ Trials assessing serelaxin (NCT01566630), pravastatin, high doses of antithrombin ${ }^{19}$ and celecoxib (NCT00442676) have been attempted, but all were terminated due to poor recruitment.

It is known that the pre-eclamptic placenta releases anti-angiogenic sFlt-1 and sEng into the maternal circulation, causing widespread maternal endothelial dysfunction and organ injury. ${ }^{20} \mathrm{~A}$ drug that can decrease sFlt- 1 and sEng production and decrease endothelial dysfunction may be a potential treatment for pre-eclampsia. We have generated preclinical data suggesting metformin may have such actions. ${ }^{9}$ Based on this preclinical data, we are now proposing to undertake this phase II randomised clinical trial.

We propose recruiting 150 women at Tygerberg Hospital in the Western Cape Province of South Africa. There are advantages to running the trial at this site. South Africa has a very high incidence of pre-eclampsia. Tygerberg Hospital is a tertiary referral centre with a neonatal intensive care unit, a maternal critical care unit and an adult intensive care unit. It is an academic centre that actively contributes to the global scientific literature and our team has recently completed the PIE trial at this research unit. We will make use of the systems set up by this trial. Thus, we believe running this trial at Tygerberg Hospital represents a balance between obtaining sufficient number of cases of preterm pre-eclampsia, performing the trial in a cost-effective manner and offering modern obstetric and perinatal care, making the results potentially generalisable to both developed and developing countries.

Metformin is known to be safe in pregnancy and is considered a category B drug in pregnancy (by the Food and Drug Administration). ${ }^{21}$ There are numerous studies of metformin for the treatment of diabetes and polycystic ovarian syndrome in pregnancy which have shown no adverse fetal effects when used in the first, second and third trimesters. ${ }^{22-27}$ Metformin does cross the placental barrier and it's long-term effect on fetal development is unknown, but a recent American College of Obstetricians 
and Gynaecologists (ACOG) practice bulletin has concluded that it can reasonably be used in pregnancy. ${ }^{28}$

In strong support of our preclinical data are meta-analyses of clinical trial data that have shown a decrease in the incidence of hypertensive disorders when metformin has been used for other indications. ${ }^{29}$ Excitingly, a randomised trial by the Fetal Medicine Foundation (for obese women without diabetes) reported that metformin significantly reduced the incidence of pre-eclampsia by as much as $76 \% .^{30}$ The EMPOWaR trial also evaluated maternal and fetal effects of metformin in obese women. ${ }^{31}$ While reporting no decrease in the incidence of hypertensive disorders of pregnancy with metformin, the most likely reason for this difference was poor compliance. ${ }^{32}$

We have powered our study for the primary outcome, which is to show whether metformin can safely prolong gestation for a further 5 days in mothers being expectantly managed for preterm pre-eclampsia. This surrogate primary outcome marker has been chosen as it provides a sample size that is feasible and attainable for a phase II study to assess efficacy. A limitation in this trial is that we have not specifically powered to detect improvements in maternal or neonatal outcomes (although these are planned secondary outcomes). If this trial yields a positive result, a further phase III multicentre randomised trial that is sufficiently powered, may be required to be sufficiently powered to demonstrate improvements in clinical outcomes.

As we did for the PIE trial, we will measure biomarkers that reflect disease severity. We will measure circulating levels of sFlt-1 and sEng, among others, in serial samples obtained from the mother. These anti-angiogenic factors are considered to play an important role in inciting maternal endothelial dysfunction and end-organ injury seen in pre-eclampsia. Furthermore, we will measure expression of key molecules in the placental samples obtained at delivery.

Pre-eclampsia kills mothers, fetuses and neonates and is responsible for severe maternal and neonatal morbidity. This is especially the case in the developing world where there is a lack of resources, including staff, equipment and finances. If a treatment were to be discovered, it would have dramatic effects on maternal and neonatal outcomes. Metformin has shown potential as a therapeutic agent in preclinical work on pre-eclampsia. Further advantages of metformin XR are that it is available in a long acting formulation, it is safe during pregnancy and it is not expensive. This makes it an ideal candidate as a global therapeutic for pre-eclampsia. It is therefore imperative for this trial to be performed. If metformin were proven to be effective at prolonging gestation in early onset pre-eclampsia it could play an important role in decreasing the clinical burden of this dangerous condition.

\section{Author affiliations}

${ }^{1}$ Department of Obstetrics and Gynaecology, Stellenbosch University Faculty of Science, Cape Town, Western Cape, South Africa
${ }^{2}$ Translational Obstetrics Group, University of Melbourne, Mercy Hospital for Women, Melbourne, Victoria, Australia

${ }^{3}$ Department of Obstetrics and Gynaecology, University of Melbourne, Mercy Hospital for Women, Melbourne, Victoria, Australia

${ }^{4}$ Department of Obstetrics and Gynaecology, School of Medicine, Monash University, Melbourne, Victoria, Australia

${ }^{5}$ Department of Obstetrics and Gynaecology, Stellenbosch University Faculty of Science, Matieland, Western Cape, South Africa

${ }^{6}$ Department of Anaesthetics, University of Melbourne, Mercy Hospital for Women, Melbourne, Victoria, Australia

${ }^{7}$ Translational Obstetrics Group and Department of Obstetrics and Gynaecology, University of Melbourne, Mercy Hospital for Women, Melbourne, Victoria, Australia ${ }^{8}$ Department of Obstetrics and Gynaecology, University of Melbourne, Melbourne, Victoria, Australia

Contributors CC, SPW and ST developed and designed the trial and obtained funding for the trial. CC, SPW and ST wrote the first draft of this manuscript. BWM and $\mathrm{DH}$ assisted with the study design. $\mathrm{RH}$ designed the statistical aspects of this protocol. FCB and TJK-L were involved in the design of the laboratory arm of this trial. All were involved in revision of the manuscript. All authors approved the final version to be submitted. CC and RH responded to the reviewer's comments.

Funding This work is supported by the Mercy Perinatal Foundation, the South African Medical Research Council and the Preeclampsia Foundation. NHMRC provides salary support to ST and BWM. The trial medication was donated by Merck pharmaceuticals.

Competing interests None declared.

Patient consent for publication Not required.

Ethics approval Stellenbosch University HREC Protocol number M16/09/037

Provenance and peer review Not commissioned; externally peer reviewed.

Open access This is an open access article distributed in accordance with the Creative Commons Attribution Non Commercial (CC BY-NC 4.0) license, which permits others to distribute, remix, adapt, build upon this work non-commercially, and license their derivative works on different terms, provided the original work is properly cited, appropriate credit is given, any changes made indicated, and the use is non-commercial. See: http://creativecommons.org/licenses/by-nc/4.0/.

\section{REFERENCES}

1. Abalos E, Cuesta C, Grosso AL, et al. Global and regional estimates of preeclampsia and eclampsia: a systematic review. Eur J Obstet Gynecol Reprod Biol 2013;170:1-7.

2. Uzan J, Carbonnel M, Piconne O, et al. Pre-eclampsia: pathophysiology, diagnosis, and management. Vasc Health Risk Manag 2011;7:467-74

3. Duley L. Perinatology S, The global impact of pre-eclampsia and eclampsia: Elsevier, 2009:130-7.

4. Khan KS, Wojdyla D, Say L, et al. WHO analysis of causes of maternal death: a systematic review. Lancet 2006;367:1066-74.

5. Zhang J, Meikle S, Trumble A. Severe maternal morbidity associated with hypertensive disorders in pregnancy in the United States. Hypertens Pregnancy 2003;22:203-12.

6. Fanaroff AA, Stoll BJ, Wright LL, et al. Trends in neonatal morbidity and mortality for very low birthweight infants. Am J Obstet Gynecol 2007;196:147.e1-147.e8.

7. Cluver CA, Walker SP, Mol BW, et al. Double blind, randomised, placebo-controlled trial to evaluate the efficacy of esomeprazole to treat early onset pre-eclampsia (PIE Trial): a study protocol. BMJ Open 2015;5:e008211.

8. Cluver CA, Hannan NJ, van Papendorp E, et al. Esomeprazole to treat women with preterm preeclampsia: a randomized placebo controlled trial. Am J Obstet Gynecol 2018;219:388.e1-388.e17.

9. Brownfoot FC, Hastie R, Hannan NJ, et al. Metformin as a prevention and treatment for preeclampsia: effects on soluble fms-like tyrosine kinase 1 and soluble endoglin secretion and endothelial dysfunction. Am J Obstet Gynecol 2016;214:356.e1-356.e15.

10. Magee LA, Pels A, Helewa M, et al. Diagnosis, evaluation, and management of the hypertensive disorders of pregnancy. Pregnancy Hypertens 2014;4:105-45.

11. Davey DA, MacGillivray I. The classification and definition of the hypertensive disorders of pregnancy. Am J Obstet Gynecol 1988;158:892-8. 
12. Hall DR. Understanding expectant management of pre-eclampsia. Obstet Gynaecol Forum 2016;26:22-7.

13. Harris PA, Taylor R, Thielke R, et al. Research electronic data capture (REDCap)-a metadata-driven methodology and workflow process for providing translational research informatics support. J Biomed Inform 2009;42:377-81.

14. Newson R. Generalized power calculations for generalized linear models and more. Stata $J$ 2004;4:379-401.

15. Hall DR, Odendaal HJ, Kirsten GF, et al. Expectant management of early onset, severe pre-eclampsia: perinatal outcome. BJOG 2000;107:1258-64.

16. Vigil-De Gracia P, Ludmir J. Perinatal and hemodynamic evaluation of sildenafil citrate for preeclampsia treatment. Obstetrics and Gynecology 2016;128:1181-2.

17. Paidas MJ, Sibai BM, Triche EW, et al. Exploring the role of antithrombin replacement for the treatment of preeclampsia: a prospective randomized evaluation of the safety and efficacy of recombinant antithrombin in very preterm preeclampsia (PRESERVE-1). Am J Reprod Immunol 2013;69:n/a.

18. Sibai B, Paidas MJ. LB02: Randomized double-blind placebo controlled evaluation of the safety and efficacy of recombinant Antithrombin versus placebo in preterm preeclampsia. Am J Obstet Gynecol 2017;216:S559-60.

19. D'Angelo A, Valsecchi L. High dose antithrombin supplementation in early preeclampsia: a randomized, double blind, placebo-controlled study. Thromb Res 2016;140:7-13.

20. Levine RJ, Maynard SE, Qian C, et al. Circulating angiogenic factors and the risk of preeclampsia. N Engl J Med 2004;350:672-83.

21. Committee T. FDA classification of drugs for teratogenic risk. Teratology society public affairs committee. Teratology 1994;49:446-7.
22. Rowan JA, Hague WM, Gao W, et al. Metformin versus insulin for the treatment of gestational diabetes. N Engl J Med 2008;358:2003-15.

23. Coetzee EJ, Jackson WP. Pregnancy in established non-insulindependent diabetics. A five-and-a-half year study at groote schuur hospital. S Afr Med J 1980;58:795-802.

24. Gilbert C, Valois M, Koren G. Pregnancy outcome after first-trimester exposure to metformin: a meta-analysis. Fertil Steril 2006;86:658-63.

25. Hughes RC, Rowan JA. Pregnancy in women with Type 2 diabetes: who takes metformin and what is the outcome? Diabet Med 2006;23:318-22.

26. Charles B, Norris R, Xiao X, et al. Population pharmacokinetics of metformin in late pregnancy. Ther Drug Monit 2006;28:67-72.

27. Lautatzis ME, Goulis DG, Vrontakis M. Efficacy and safety of metformin during pregnancy in women with gestational diabetes mellitus or polycystic ovary syndrome: a systematic review. Metabolism 2013;62:1522-34.

28. ACOG Practice Bulletin. ACOG practice bulletin No. 190. Obstetrics \& Gynecology 2018;131:e49-64.

29. Gui J, Liu Q, Feng L. Metformin vs insulin in the management of gestational diabetes: a meta-analysis. PLoS One 2013;8:e64585.

30. Syngelaki A, Nicolaides $\mathrm{KH}$, Balani J, et al. Metformin versus placebo in obese pregnant women without diabetes mellitus. N Engl J Med 2016;374:434-43.

31. Chiswick C, Reynolds RM, Denison F, et al. Effect of metformin on maternal and fetal outcomes in obese pregnant women (EMPOWaR): a randomised, double-blind, placebo-controlled trial. Lancet Diabetes Endocrinol 2015;3:778-86.

32. Romero R, Erez O, Hüttemann M, et al. Metformin, the aspirin of the 21st century: its role in gestational diabetes mellitus, prevention of preeclampsia and cancer, and the promotion of longevity. Am J Obstet Gynecol 2017;217:282-302. 